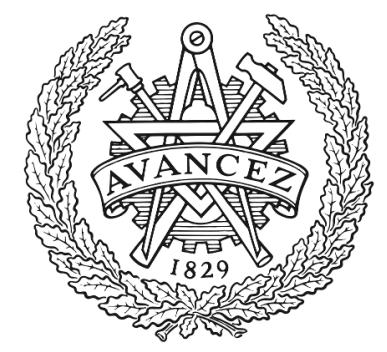

CHALMERS

UNIVERSITY OF TECHNOLOGY

\title{
Large-Signal Circuit Model for Datacom VCSELs
}

Downloaded from: https://research.chalmers.se, 2023-04-26 07:58 UTC

Citation for the original published paper (version of record):

Grabowski, A., Gustavsson, J., He, Z. et al (2018). Large-Signal Circuit Model for Datacom VCSELs. Conference Digest - IEEE International Semiconductor Laser Conference, 2018-September: 193-194. http://dx.doi.org/10.1109/ISLC.2018.8516232

N.B. When citing this work, cite the original published paper. 


\title{
Large-signal circuit model for datacom VCSELs
}

\author{
Alexander Grabowski ${ }^{1, *}$, Johan Gustavsson ${ }^{1}$, Zhongxia Simon $\mathbf{H e}^{2}$, and Anders Larsson ${ }^{1}$ \\ ${ }^{1}$ Photonics Laboratory, MC2, Chalmers University of Technology, SE-41296, Gothenburg, Sweden \\ ${ }^{2}$ Microwave Electronics Laboratory, MC2, Chalmers University of Technology, SE-41296, Gothenburg, Sweden \\ *alexander.grabowski@chalmers.se
}

\begin{abstract}
We present a physics-based equivalent-circuit model for datacom VCSELs, with ambient temperature and self-heating effects accounted for. This circuit model is unique in accounting for carrier capture dynamics between active region continuum and quantum well bound states. OOK/PAM4 simulations are demonstrated at $25^{\circ} \mathrm{C}$ and $85^{\circ} \mathrm{C}$.
\end{abstract}

\section{INTRODUCTION}

VCSELs are highly appealing to the datacom industry for several reasons, including low energy consumption per bit and volume manufacturing capabilities. Today, datacom VCSEL and detector bandwidths are limited to roughly $30 \mathrm{GHz}$ [1], and the development of next generation VCSEL-based optical interconnects operating at $\geq 56 \mathrm{~Gb} / \mathrm{s}$ single lane rates require more sophisticated driver and receiver electronics. This can for example be achieved using pre-emphasis and equalization, as demonstrated in [2]. But in order to optimize performance and minimize power consumption for these more complex optical interconnects it becomes essential to co-optimize the driver and receiver electronics with the VCSEL. Therefore, there is a need of a simple yet accurate large-signal VCSEL model, preferably a circuit-level based one, which can predict important characteristics such as impedance mismatch, optical output power saturation, and modulation speed limitations. As many VCSEL properties are temperature dependent, resulting from the strong self-heating in the device and ambient temperature varying with application, the model must include temperature effects. Therefore, in this work we have developed a circuit-level model for datacom VCSELs, where the internal device temperature is tracked via a sub-circuit and the device parameters are made temperature dependent. Examples of similar works on this topic can be found in [3], [4] and [5]. What distinguishes this work from the previous ones is the usage of two sub-circuits for keeping track of the free carriers in the continuum and quantum well bound states of the active region, respectively. This carrier transport effect is well-known to have an impact on the modulation bandwidth of high-speed semiconductor lasers [6].

\section{Circuit MODEL}

Our equivalent circuit VCSEL model consist of five interdependent sub-circuits, as can be seen in Figure 1. The first sub-circuit models the device input impedance and the voltage drop over intrinsic region. The second and third sub-circuits model the free carrier accumulation in the continuum and quantum wells, respectively, of the active region under current injection. In this way, the important carrier capture dynamics from continuum to quantum well bound states can be accounted for. The fourth sub-circuit models the photon accumulation in the optical resonator from stimulated emission, where a fraction of these photons escape through the top mirror to produce the useful output power. The fifth sub-circuit is used to track the internal device temperature via a thermal impedance model, accounting both for self-heating and ambient temperature effects. The model is implemented in Advanced Design System (ADS), and is in this work operating under the device parameters of a $7 \mu$ m oxide aperture $850 \mathrm{~nm}$ datacom VCSEL. The model is physics-based utilizing a conventional rate equation description of semiconductor lasers, where only extracted physical properties from measurements and theoretical calculations are used. Thus, it is not an empirical model, meaning that there is no direct fitting of simulated to measured data. Comparison of simulated to measured data is yet to be done, as work with extracting more accurate values of some device parameters is still in progress.

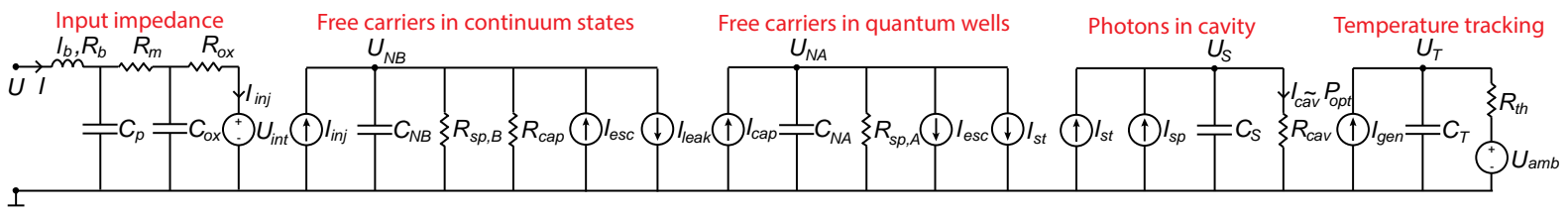

Fig. 1. The five sub-circuits forming the total circuit-level VCSEL model.

\section{SIMULATIONS}

Examples of simulated VCSEL static characteristics can be seen in Figure 2 (a-c). Subfigures a) and b) show steady-state L-I-V characteristics for a temperature interval of $25^{\circ} \mathrm{C}$ to $95^{\circ} \mathrm{C}$ with a step of $10^{\circ} \mathrm{C}$. Subfigure c) shows that we can track the internal temperature with respect to input current, and from this we are also able to determine the relation between the input current and the resonance wavelength.

In order to determine the VCSEL small-signal direct-modulation response we can simulate the circuit scattering parameters S11 and S21, which are both relevant for VCSELs in terms of impedance and throughput parameters such as bandwidth. Figure 3 (a-b) shows examples of simulated S11- and S21-responses respectively. 

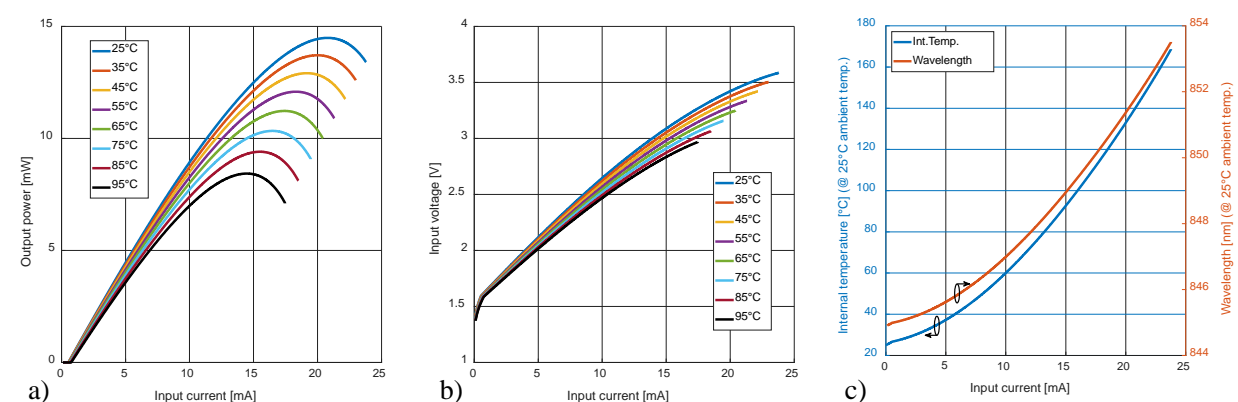

Fig. 2. a) Optical output power vs input current. b) Input voltage vs input current. c) Internal temperature and wavelength vs input current

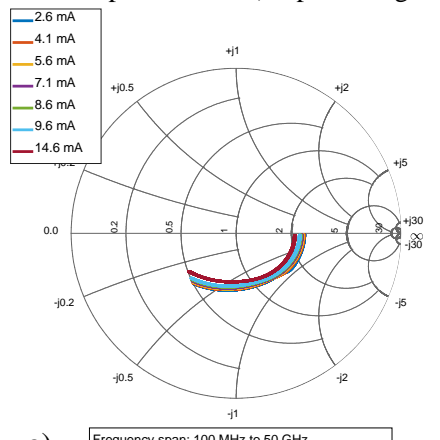

a)

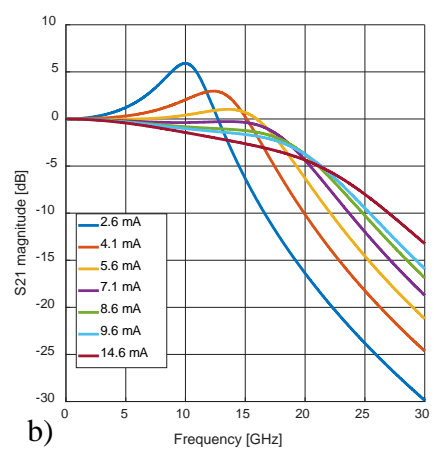

Fig. 3. a) Simulated S11-response $\left(25^{\circ} \mathrm{C}\right)$. b) Simulated S21-response $\left(25^{\circ} \mathrm{C}\right)$, offset to $0 \mathrm{~dB}$ at starting frequency.

For large-signal simulations we have varied both modulation rate and ambient temperature. We also demonstrate the capability of the model to simulate PAM-4 transmission. This is purely a back-to-back simulation not including any photodiode or receiver electronics. The transmitter is a $50 \Omega$ signal generator with implemented rise time of roughly one-fifth of a bit slot, thus it is not in any way impedance matched to the VCSEL model. The simulation examples can be seen in Figure 4 (a-d), in which PRBS7 was used and the output was AC-coupled.
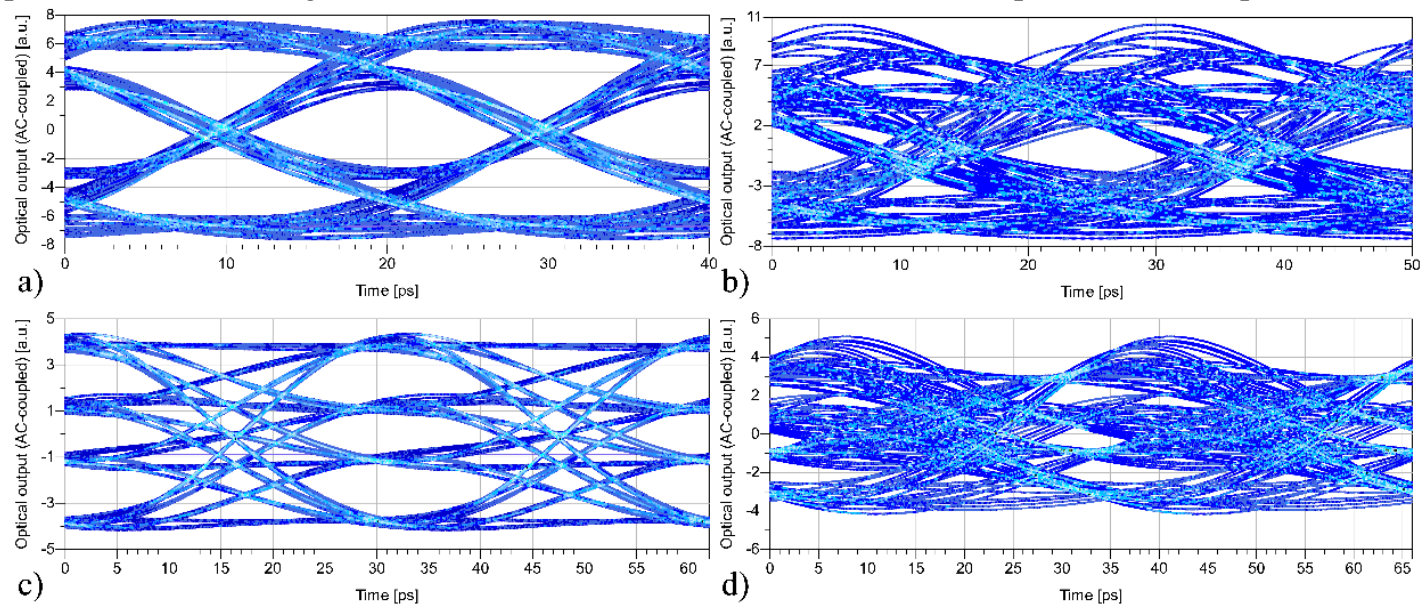

Fig. 4. a) Simulated OOK at $50 \mathrm{Gbps}, 25^{\circ} \mathrm{C}, 10 \mathrm{~mA}$ bias. b) Simulated $\mathrm{OOK}$ at $40 \mathrm{Gbps}, 85^{\circ} \mathrm{C}, 6 \mathrm{~mA}$ bias

c) Simulated PAM-4 at 32 Gbaud, $25^{\circ} \mathrm{C}, 10 \mathrm{~mA}$ bias. d) Simulated PAM-4 at $30 \mathrm{Gbaud}, 85^{\circ} \mathrm{C}, 6 \mathrm{~mA}$ bias.

\section{CONCLUSION}

We have developed a physics-based circuit model for datacom VCSELs capable of recreating static, small-signal and large-signal characteristics. Measurements for comparison are yet to be done.

\section{REFERENCES}

[1] E. Haglund et al., "30 GHz bandwidth $850 \mathrm{~nm}$ VCSEL with sub-100 fJ/bit energy dissipation at 25-50 Gbit/s," Electron. Lett., vol. 51, no. 14, pp. 1096-1098, Jul. 2015.

[2] J. Lavrencik, V. A. Thomas, S. Varughese, and S. E. Ralph, “DSP-Enabled 100 Gb/s PAM-4 VCSEL MMF Links,” J. Light. Technol., vol. 35, no. 15, pp. 3189-3196, Aug. 2017.

[3] P. V. Mena, J. J. Morikuni, S.-M. Kang, A. V. Harton, and K. W. Wyatt, "A comprehensive circuit-level model of vertical-cavity surface-emitting lasers,” J. Light. Technol., vol. 17, no. 12, pp. 2612-2632, 1999.

[4] P. V. Mena, J. J. Morikuni, S.-M. Kang, A. V. Harton, and K. W. Wyatt, “A simple rate-equation-based thermal VCSEL model,” J. Light. Technol., vol. 17, no. 5, pp. 865-872, May 1999.

[5] K. Szczerba, C. Kocot, and G. Landry, "A Simple Circuit-Level Model of Vertical-Cavity Surface-Emitting Lasers Working Over a Broad Temperature Range,” in 2017 European Conference on Optical Communication (ECOC), 2017, no. 1, pp. 1-3.

[6] L. A. Coldren, S. W. Corzine, and M. L. Mašanović, Diode Lasers and Photonic Integrated Circuits, 2nd ed., vol. 218. Hoboken, NJ, USA: John Wiley \& Sons, Inc., 2012. 